\title{
LA COLECCIÓN DE BATRACOLOGÍA Y HERPETOLOGÍA DEL MUSEO NACIONAL DE CIENCIAS NATURALES (CSIC)
}

\author{
J. E. González Fernández (*)
}

\begin{abstract}
RESUMEN
La Colección de Batracología y Herpetología del Museo Nacional de Ciencias Naturales, con más de doscientos años de antigüedad, está compuesta por cerca de 40.000 ejemplares, constituyendo la mayor colección de España en su materia y, sin duda, la mejor y más representativa de fauna ibérica de anfibios y reptiles del mundo. Posee representación de 273 especies de anfibios y 473 de reptiles, agrupadas en 25 y 33 familias respectivamente. Aunque mayoritariamente ibéricos, existen muestras significativas de la fauna europea, y de aquellos otros continentes en los que en el pasado España tuvo responsabilida política. Posee una importante colección de ejemplares y series tipo, tanto de especies españolas, como sudamericanas, africanas o asiáticas. La colección está inventariada en el $60 \%$ e informatizada en su 45\%. Existen diversas "Colecciones de autor", desde el material procedente de la colección inicial de Franco Dávila, pasando por las de Graells, Boscá, Jiménez de la Espada, etc. Por su carácter público, su utilización está abierta a toda la comunidad científica, constituyendo también el lugar ideal para el depósito y conservación de los ejemplares procedentes de la investigación científica, sobre todo los ejemplares tipo, por contar con un presupuesto estable para su conservación y mantenimiento.
\end{abstract}

Palabras clave: Colecciones, anfibios, reptiles, conservación.

\section{ABSTRACT \\ The Collection of Batrachology and Herpetology of the Spanish Museo Nacional de Ciencias Naturales (CSIC)}

The Collection of Batrachology and Herpetology of the Spanish Museo Nacional de Ciencias Naturales, has more than two hundred years old, contain about 40.000 specimens. It's the biggest collection of his type in Spain and also the best and the most representive of iberian fauna in the word. It has representation of 273 anfibians species and 473 of reptiles, grouped into 25 and 33 families respectively. The most of them are spanish, but there are significant samples of the european fauna, and faunas of others continents where Spain has politics influence in the past. It's has an importan collection of type specimens and series, as spanish species as sudamerican, african or asiatic specimens. The collection is inventory in $60 \%$ and computerized in $45 \%$. There are several "Author collections", since a few initials specimens of Franco Davila collection, passing for Graells', Boscá's, Jiménez de la Espada's collections, and so on. It is a public collection with an annual estimate for management and conservation, for that it can by used by all scientific comunity, also is an ideal institution to placed and to preserve the specimens coming from scientic research, overcoat type specimens.

Key words: Collections, amphibians, reptiles, preservation.

* Colección de Batracología y Herpetología. Vicedirección de Colecciones y Documentación. Museo Nacional de Ciencias Naturales, C.S.I.C. José Gutiérrez Abascal, 2, 28006 Madrid. 


\section{Introducción histórica}

La colección de Batracología y Herpetología del Museo Nacional de Ciencias Naturales (MNCN) tiene su origen en el Gabinete de D. Pedro Franco Dávila, adquirido por Carlos III a finales de 1771 (Barreiro, 1992).

Como primer hito a destacar deberíamos reseñar la creación en 1819 de la Cátedra de Anatomía Comparada, ocupando la misma D. Tomás Villanova. En 1833 se nombra conservador del Museo, a D. Eduardo Villanova, quien para ello presenta como uno de sus méritos, la realización del "CATÁLOGO DE LOS REPTILES DEL MUSEO", que posiblemente fue el primer inventario que se hiciere, un manuscrito inédito, que desafortunadamente no se conserva en la actualidad, y que probablemente nunca vio la luz como una publicación por la desgraciada muerte de D. Eduardo Villanova, seis meses más tarde. En 1835 regresa de Cuba D. Ramón de la Sagra, con material herpetológico colectado por él entre 1823 y 1835 así como el colectado por el Dr. Poey.

Tras un periodo de casi cuarenta años, en 1875 D. Marcos Jiménez de la Espada finaliza el estudio de parte del material colectado por la Comisión Científica al Pacífico (1862-1865) y publica la muy conocida monografía: "Vertebrados del Viaje al Pacífico, BATRACIOS". En 1880 ingresan en el Museo los ejemplares colectados por la Comisión Científica del Pacífico que permanecían en el Real Jardín Botánico.

E. Boscá dona entre 1879 y 1882 , entre otros, los ejemplares TIPO de las especies ibéricas de anfibios y reptiles hasta entonces por él descritas. En 1914 tiene lugar la donación de E. Boscá de parte de su valiosa colección de anfibios y reptiles.

A principios de 1900 ingresan los ejemplares recolectados en Siria y Persia por los hermanos M. y F. Martínez de la Escalera. Desde 1905 y hasta 1910 se sucenden los ingresos procedentes del Noroeste Africano depositados por la Real Sociedad de Historia Natural.

Durante los primeros años de la década de los treinta, la colección es revisada por E. Cusi, aunque no da lugar a publicación de catálogo alguno.

La guerra civil abre un paréntesis en la actividad de la sección de Zoología del MNCN, que desgraciadamente se prolongará durante décadas.

A partir de 1981 se crean nuevas colecciones de esqueletos desarticulados y de esqueletos teñidos y articulados. Una mayor atención institucional dota a las colecciones de algún personal y medios, y se modernizan las instalaciones del Museo, lo que permite tras muchos años, que comienze a dar servicio a la comunidad científica.

De reciente ingreso en el MNCN, por donación de la Comunidad Autónoma de Madrid (CAM), cabe destacar la colección de la desaparecida Unidad de Zoología Aplicada (UZA) que supone una notable incorporación a la colección ya existente. El procesado de dicho material, se encuentra actualmente en curso.

\section{Composición numérica, taxonómica y geográfica}

Compuesta por unos 40.000 ejemplares (25.000 anfibios y 15.000 reptiles), constituye la mayor colección de España en su materia y, sin duda, la mejor y más representativa de fauna ibérica de anfibios y reptiles del mundo.

La mayor parte de ellos, cerca del 90\%, se conservan completos en fluido (soluciones acuosas de etanol y formaldehído) y el resto son esqueletos desarticulados o teñidos y transparentados. También exite una pequeña colección de unos 250 ejemplares naturalizados, la mayoría de ellos provenientes de los siglos XVIII y XIX.

Aunque por el volumen total, unos 4.000 ejemplares (el 10\% del total) pudiera parecer poco significativo, hay que destacar la importancia de la colección de esqueletos, muy especialmente del conjunto de especímenes que se encuentran teñidos selectivamente y transparentados. Es esta la mejor colección de su clase de las existentes en España y de las mejores de Europa, sin competencia en lo que a fauna ibérica se refiere (González-Fernández, 1996)

En lo que atañe al material inventariado, en la colección existen representantes de unas 700 especies de herpetos, pertenencientes a 58 familias, 25 de anfibios (Ambystomatidae, Arthroleptidae, Ascaphidae, Bufonidae, Caecilidae, Cetrolenidae, Cryptobranchidae, Dendrobatidae, Discoglossidae, Hylidae, Hyperolidae, Ichtyophidae, Leptodactylidae, Microhylidae, Pelobatidae, Pelodytidae, Pletodontidae, Proteidae, Pipidae, Ranidae, Rhacophoridae, Rhinodermatidae, Rhinophrynidae, Salamandridae y Typlonectidae) y 33 de reptiles (Acrochordidae, Agamidae, Anguidae, Aniliidae, Alligatoridae, Amphisbaenidae, Bipedidae, Boidae, Chamaeleonidae, Chelidae, Cheloniidae, Colubridae, Cordylidae, Crocodylidae, Crotalidae, Elapidae, Emydidae, Gekkonidae, Hidrophiidae, Iguanidae, 
Kinosternidae, Lacertidae, Laticaudidae, Leptotyphlopidae, Pelomedusidae, Scincidae, Teidae, Testudinidae, Trionychidae, Trogonophidae, Varanidae, Viperidae, Xantusiidae) incluidas en 8 órdenes (Amphisbaenia, Anura, Caudata, Crocodylia, Gymnophiona, Lacertilia, Serpentes y Testudines) (Tabla 1).

Tabla 1.—Número de especies (ESP.) y familias (FAM.) representadas por órdenes.

\begin{tabular}{llrr} 
CLASE & ORDEN & $\mathbf{N}^{\circ}$ ESP. & $\mathbf{N}^{\circ}$ FAM. \\
\hline AMPHIBIA & & $(273)$ & \multicolumn{2}{c}{$(25)$} \\
& ANURA & 211 & 17 \\
& CAUDATA & 53 & 5 \\
& GYMNOPHIONA & 9 & 3 \\
REPTILIA & & $(473)$ & $(33)$ \\
& AMPHISBAENIA & 9 & 3 \\
& CHELONIA & 31 & 7 \\
& CROCODILIA & 8 & 2 \\
& LACERTILIA (= SAURIA $\left.{ }^{*}\right)$ & 265 & 11 \\
& SERPENTES $\left(=\right.$ OFIDIA $\left.^{*}\right)$ & 160 & 10 \\
\hline TOTALES & & $\mathbf{( 7 4 6 )}$ & $\mathbf{( 5 8 )}$ \\
\hline
\end{tabular}

La representación geográfica de la parte informatizada de la colección es fundamentalmente ibérica, aunque existen muestras significativas del resto de la fauna europea y de aquellos otros lugares donde España tuvo responsabilidad política en tiempos pasados (Figura 1).

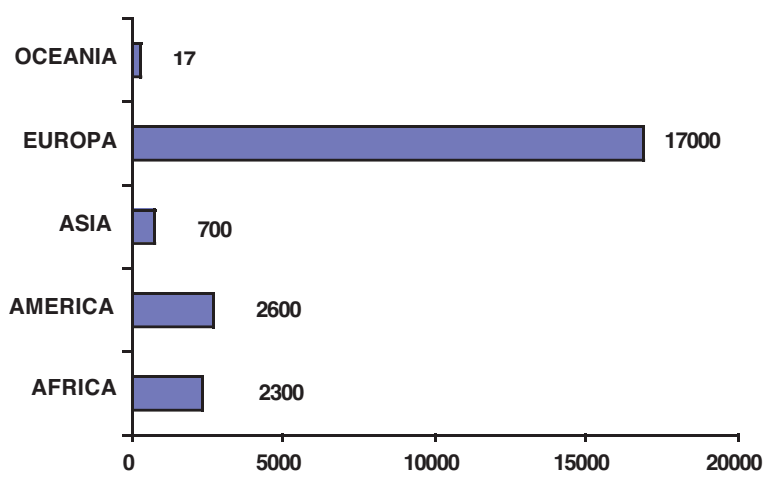

Fig. 1.- Histograma por continentes.

\section{Colección de tipos}

Al hacer una panorámica sobre la composición de la colección, no podemos olvidar uno de los aspectos más preciados y valorados de toda colección científica, cual es la existencia de EJEMPLARES TIPO o pertenecientes a SERIES TÍPICAS.

En este sentido la colección de Batracología y Herpetología del MNCN, es afortunada y rica al mismo tiempo, ya que desde los tipos de las especies descritas por Jiménez de la Espada, procedentes del material colectado por la Comisión Científica del Pacífico, y en parte recuperados en estos últimos años, hasta los de los Doctores J. Serra-Cobo (1992), M. García-París (1995) y M. Vences (1997) pasando por las series de E. Boscá, J.M ${ }^{a}$ Valverde, A. Salvador, S.D. Busack y C.E. Peréz-Santos, son casi una cuarentena los taxones de cuyo material típico tenemos representación, siendo más de 300 el número de ejemplares pertenecientes a estas series típicas (Tabla 2).

Tabla 2.- Material típico recuperado existente en la Colección.

\begin{tabular}{lcc} 
AUTOR & $\begin{array}{c}\text { TAXONES } \\
\text { DESCRITOS }\end{array}$ & $\begin{array}{c}\text { TAXONES } \\
\text { LOCALIZADOS }\end{array}$ \\
\hline Jiménez de la Espada & 41 & 20 \\
Boscá & 5 & 2 \\
Boulenger & 5 & 0 \\
Valverde & 1 & 1 \\
Salvador & 11 & 11 \\
Busack & 1 & 1 \\
Peréz-Santos & 1 & 1 \\
Serra-Cobo & 1 & 1 \\
García-París & 3 & 3 \\
\hline
\end{tabular}

\section{Estado del inventario y grado de informatización}

De los cerca 40.000 ejemplares que comprende el total de la colección, se encuentran revisados e inventariados unos 25.000, de los cuales están informatizados alrededor 23.000 , estando los 15.000 restantes están en una fase preliminar de revisión e inventario (Tabla 3 ). 
Tabla 3.- Estado del inventario e informatización por órdenes.

\begin{tabular}{llrrr} 
CLASE & ORDEN & INVENTARIADO & INFORMATIZADO & PENDIENTE \\
\hline AMPHIBIA & & 16.400 & 16.400 & 8.000 \\
& ANURA & 13.000 & 13.000 & 6.000 \\
& CAUDATA & 3.475 & 3.475 & 2.000 \\
REPTILIA & GYMNOPHIONA & 25 & 25 & 0 \\
& & 8.600 & 6.600 & 7.000 \\
& AMPHISBAENIA & 130 & 130 & 100 \\
& CHELONIA & 220 & 220 & 50 \\
& CROCODILIA & 20 & 20 & 0 \\
& OPHIDIA & 1.230 & 3.230 & 3.000 \\
& SAURIA & 5.000 & 5.000 & 3.850 \\
\hline TOTALES & & $\mathbf{2 5 . 0 0 0}$ & $\mathbf{2 3 . 0 0 0}$ & $\mathbf{1 5 . 0 0 0}$ \\
\hline
\end{tabular}

\section{Colecciones de autor}

La Colección de Batracología y Herpetología se encuentra dividida por el caracter taxonómico en los dos grandes grupos zoológicos que la componen, los anfibios y los reptiles. Las otras subdivisiones tienen que ver con el tipo de conservación que afecta a los ejemplares: naturalizados, en fluido y esqueletos.

Pese a que no existan divisiones físicas, por los autores o colectores, ni tan siquiera por la procedencia, sí que podemos distinguir diferentes "subcolecciones" atendiendo al origen de los ejemplares.

1.- Colección Franco Dávila. Pocos son los ejemplares que a ciencia cierta podemos asegurar que pertenecieron a la colección original del Museo, sin embargo alguno de los cocodrilos naturalizados seguramente proceden de esta época por las indicaciones de J.B. Bru, primer pintor y disecador del Gabinete (Bru, 17841786).

2.- Colección Antonio Parra. Este naturalista envía entre 1788 y 1793 diverso material zoológico colectado en la isla de Cuba. Ocurre algo similar al caso anterior, sin embargo se conserva una tortuga naturalizada (Caretta caretta) que presenta "Balanos" en su concha, circunstancia recogida ya por J. Mieg (Mieg, 1818) y que nos indica su más que probable procencia de esa época.

3.- Colección Graells. Nombrado Graells catedrático de Anatomía Comparada en 1838, impulsa bajo su dirección la confección por parte de los disecadores del Museo, de una serie de esquele- tos montados y ejemplares naturalizados, que servían para la enseñanza de la asignatura. De esta época se conservan diversas piezas montadas, obra de Duchen (un esqueleto de galápago Mauremys leprosa de 1844, otro de camaleón Chamaeleo chamaeleon de 1850 , etc.).

4.- Colección del Pacífico, confeccionada a partir del material que la Comisión Científica del Pacífico colectó en su viaje por el continente americano entre 1862 y 1865 . Conocida también como colección Jiménez de la Espada, por haber sido dicho miembro de la Comisión, el encargado del estudio de la misma, es sin duda la "subcolección" más importante de todas por diferentes motivos:

A.- Incluye el 95\% del material americano existente en la colección

B.- A ella pertenece el $50 \%$ del material TIPO de la colección.

C.- Los estudios de M. Jiménez de la Espada basados en esta colección, son aún referencia para todo estudio de la fauna Batracológica y Herpetológica neotropical. No sólo incluye material conservado en fluido, sino también deferentes ejemplares naturalizados, como la piel de un caimán (Melanosochus niger) que se exhibe en la actualidad a la entrada de la exposición histórica.

5.- Colección E. Boscá. Catedrático de Instituto en sus inicios y finalmente director del valenciano Museo Botet de Paleontología, E. Boscá es considerado como el padre de la Herpetología ibérica. A él se debe la descripción de muchas especies españolas como Vipera latastei (Boscá, 1879a), Alytes cisternasii (Boscá, 1879b), Hyla 
perezi (Boscá, 1880a), Chalcides bedriagai (Boscá, 1880b), y Lacerta pityusensis (Boscá, 1882), algunos de cuyos ejemplares TIPO se conservan en el Museo. Igualmente se conserva su colección, fundamentalmente ibérica, donada en 1914.

6.- Colección A. Salvador, donada cuando dicho investigador se incorporó al Museo a finales de los ochenta. Constituye una importante y cuidada representación de fauna ibérica bien documentada. Está enriquecida por la aportación de un número importante de ejemplares tipo de diversas subespecies de lagartijas de Baleares.

7.- Colección de la Comunidad Autónoma de Madrid, procedente de la antigua Unidad de Zoología Aplicada, dependiente inicialmente del ICONA y posteriormente del INIA. Esta colección está formada por entre trece y quince mil ejemplares, y constituye la más reciente y abundante aportación a las colecciones del Museo. De origen fundamentalmente ibérico, y aunque en desigual estado de conservación y datación, se encuentra en la actualidad en proceso de inventario.

8.- Colección Pérez Santos. Donada el museo entre 1997 y 1998 esta constituida por unos 600 ejemplares de Fauna Neotropical, fundamentalmente ofidios, pertenecientes a los paises de Ecuador Colombia y Panamá. Aunque no muy elevada en número, es importante por varios motivos:

A.- Representa la primera aportación "masiva" de ejemplares de fauna neotropical, desde la Comisión Científica del Pacífico (18621865).

B.- Se encuentra en muy buen estado de conservación y correctamente datada y determinada.

C.- Muchos de los ejemplares que la forman, han sido utilizados para la confección de los libros "Los ofidios de Colombia" y "Los Ofidios de Ecuador".

\section{Catálogos publicados}

En 1833 D. E. Villanova accede a la plaza de Conservador del Museo, por ser poseedor entre otros méritos del de haber confeccionado "EL CATÁLOGO DE LOS REPTILES DEL MUSEO", desgraciadamente no se conserva ninguna copia de dicho manuscrito en los archivos del Museo, quizás por su trágica muerte acaecida seis meses más tarde de su toma de posesión.
En 1838 la Junta Gubernativa nombra catedrático de Anatomía comparada a M. P. Graells, y a él se deben entre otras muchas cosas el catálogo manuscrito de 1846, el más antiguo que ha llegado hasta nosotros. En este catálogo encontramos referencia de los ejemplares conservados en fluido mas antiguos de la colección, se trata de dos ranas verdes (Rana perezi) procedentes de Logroño (La Rioja).

Después de esta fecha no se conocen más textos genéricos publicados o manuscritos sobre el total de la colección. Existen ciertos trabajos relacionados con diversos aspectos parciales, como la obra ya mencionada de M. Jiménez de la Espada (1875) o el libro de J. Gogorza (Gogorza, 1891).

Ya dentro de este siglo, han existido varias revisiones de la colección, la primera de ellas la efectuada por Antonio de Zulueta, en los años anteriores a la primera guerra mundial, y la de Ernesto Cusi en los años treinta, de ninguna de ellas ha quedado ninguna constancia en libros o catálogos manuscritos o publicados, sólo las anotaciones en tal sentido efectuadas en las fichas antiguas de la colección.

\section{Disponibilidad de la colección}

La colección de Batracología y Herpetología del MNCN, por su carácter público, se encuentra abierta para su utilización a toda la comunidad científica, existiendo personal técnico encargado del mantenimiento y gestión de los fondos en ella depositados.

Para su uso correcto, existe una normativa de colecciones y unas condiciones de manipulación en los préstamos y consultas (Sanchiz, 1994).

Como ya hemos indicado anteriormente, la colección se encuentra informatizada en un alto porcentaje, inicialmente gracias al proyecto "Museología del Patrimonio Natural" dirigido por el Dr. B. Sanchiz; perfeccionado posteriormente por el conservador de la colección, quien desarrolló un programa en Dbase IV (González-Fernández, 1994). Gracias a ése programa, el acceso a la información que sobre los 23.000 ejemplares informatizados poseeemos es rápida, ágil y eficaz. En la actualidad se esta transformando a ACCESS, con el objeto de mejorarlo y hacerlo compatible con otras bases de datos geográficas, taxonómicas, etc., con objeto de mejorar la información ofrecida al usuario.

Para utilizar la colección es necesario solicitar la consulta o el préstamo del material al conservador, acompañando la demanda (en el caso de estudiantes o recién graduados sin "curriculum" investiga- 
dor) de un aval científico del investigador que le dirige. La solicitud debe hacerse con un breve periodo de antelación, en el caso de las consultas. Los préstamos se realizan en las mismas condiciones, por periodos de seis meses renovables.

Por contar con técnicos y estar dotada de un presupuesto anual estable que asegura su gestión y mantenimiento, la coleción del MNCN, es el lugar idóneo para el depósito de los ejemplares existentes en colecciones privadas o públicas de menor entidad, de alto costo de mantenimiento, para la salvaguarda de ejemplares tipo, o de los utilizados en las investigaciones científicas. Fruto de este criterio, ha sido la donación al Museo de los holotipos de todas las especies recientemente descritas de la fauna batracológica ibérica, por parte de los correspondientes equipos de investigación.

Los responsables de la colección, también puede prestar asesoramiento en el mantenimiento, gestión, conservación y mantenimiento de pequeñas colecciones.

No resta sino indicar a todo aquel interesado en la Batracología y Herpetología, la dirección de contacto:

José E. González Fernández

(Colección de Batracología y Herpetología)

Museo Nacional de Ciencias Naturales

C/ José Gutiérrez Abascal, 2

28006 Madrid (ESPAÑA)

Telf.: 914.11.13.28 ext.1122

fax: 34 (9) 5645078

e-mail: JEGONZALEZ@MNCN.CSIC.ES

\section{AGRADECIMIENTOS}

En primer lugar agradecer a la "Revista Graellsia", representada por su comité editorial, la oportunidad brindada para dar a conocer mejor la colección de Batracología y Herpetología del Museo. También agradezco la colaboración prestado por el técnico preparador del Museo D. Francisco Yagüe, en las tareas de inventariado y preparación de ejemplares.

Igualmente quiero mostrar mi agradecimiento a la Sociedad de Amigos del Museo Nacional de Ciencias Naturales, a través de la cual, y como voluntarios de colecciones, han colaborado desinteresadamente diferentes personas, inventariando o informatizando registros. Por su especial dedicación y desinterés, es de justicia mencionar entre todas ellas a las siguientes: Sofía Escolar Huete, Alicia González Cabeza, Lidia Hernández Moraleda, Juan Carlos Peracho Guevara, Áurea Sevilla de la Torre y Leonor Zarza Caballero. A las cuales desde aquí les deseo los mayores éxitos en su carrera profesional.

Finalmente sería imperdonable no manifestar mi agradecimiento a aquellas personas que áun, no siendo Biólogos, han colaborado con las tareas desarrolladas en la colección de
Batracología y Herpetología realizando La Prestación Social Sustitutoria en la misma: Luis Duque Duque, David Guillamón Fernández, Javier D. Fernández Alonso y Raúl Sánchez de Pablos.

\section{Referencias}

Barreiro, A.J., 1992. El Museo Nacional de Ciencias Naturales (1771-1935). Museo Nacional de Ciencias Naturales \& Ediciones Doce Calles. Madrid. 509 pp.

BRU, J.B., 1784. Colección de láminas que representan a los animales y monstruos del real Gabinete de Historia Natural. 2 Tomos. Imprenta de Andrés de Sotos. Madrid.

GogorzA, J., 1891 Reseña y Guía de las Colecciones del Museo de Historia Natural . Escuela Tipográfica del Hospicio. Madrid. 103 pp.

GonZÁlez-FernándeZ, J.E., 1994. Programa Ireneo, una solución a la informatización de coleciones de Herpetología. Abstracts III Congreso Luso-Español, VII Español de Herpetología, Badajoz :58.

GONZÁLEZ-FERnÁNDEZ, J.E., 1996. Los esqueletos en la colección de herpetología del MNCN. La subcolección de esqueletos teñidos. IV Congreso LusoEspañol y VII Congreso Español de Herpetologia, Oporto: 48.

Graells, M. DE LA P., 1846. Catálogos de las colecciones de Anatomía Comparada, Mamíferos, Aves, reptiles y Peces del Museo Nacional de Ciencias Naturales de Madrid. Manuscrito, Inédito, Madrid.

JiméneZ DE la EsPADA, M., 1875. Vertebrados del Viaje al Pacífico. Tomo I: Batracios. Imprenta de Miguel Ginesta. Madrid. 208 pp y 7 lám.

Mieg, J., 1818. Paseo por el Gabinete de Historia Natural de Madrid. Imprenta de D.M. de Burgos. Madrid. Tomo I: 512 pp, Tomo II: 15 lám.

SAnchiz, B., (ed.) 1994. Manual de Catalogación y Gestión de las Colecciones Cientificas de Historia Natural. Museo Nacional de Ciencias Naturales, CSIC. Madrid. 238 pp. 